\title{
Report from the 4th annual Society of Cardiac Computed Tomography Japan Forum
}

\author{
Frank J. Rybicki · Sachio Kuribayashi
}

Received: 17 September 2010/ Accepted: 23 September 2010/Published online: 6 October 2010

(C) Springer Science+Business Media, B.V. 2010

The 4th annual meeting of the Japan Regional Committee of the Society of Cardiovascular Computed Tomography featured developments and implementation of new algorithms for multidetector CT. The Japan Regional Committee is acknowledged as the first organized international regional committee of this society. The meeting had 284 attendees and was held at Shinagawa in Tokyo, Japan on September 4th, 2010. The program featured talks focusing on high quality, reproducible, and accurate coronary CT angiography. For example, in the lecture titled "Radiation Dose Reduction for 320-row CT" by Dr. Masatoshi Tsunoda, protocol strategies to reduce radiation and iodinated contrast media were detailed. With respect to radiation, there was a large experience in imaging on the order of $2 \mathrm{mSv}$ or less, with lower limits on the order of $0.5 \mathrm{mSv}$ for a complete coronary CT angiogram. To lower radiation, different institutions used

Core 320 Participating Sites (Principal Investigator). Iwate Medical University, Dr. Tetsuya Fusazaki; Keio University, Dr. Sachio Kuribayashi; Mie University, Dr. Hajime Sakuma; St. Luke's International Hospital, Dr. Hiroyuki Niinuma.

\section{F. J. Rybicki (凹)}

Applied Imaging Science Laboratory, Noninvasive Cardiovascular Imaging Program, Brigham and Women's Hospital \& Harvard Medical School, Radiology, 75 Francis Street, Boston, MA 02115, USA e-mail: frybicki@partners.org

S. Kuribayashi

Keio University School of Medicine, Tokyo, Japan iterative reconstruction, phase shift reconstruction, and volume scan techniques. Lower-dose coronary CTA and important clinical applications were featured in original articles within a recent edition of the International Journal of Cardiovascular Imaging [1].

The Japan Regional Committee also featured "Skill-up in Cardiac CT through e-Learning", an educational report given via teleconference by Dr. Yasushi Koyama. This project introduced a new Japanese web-based e-learning system. This database is comprised of 650 cases, each with a set of questions and answers accessed online. This method of learning is unique in Japan and was met with great enthusiasm among the participants.

Although difficult to obtain absolute numbers, Japan has one of, if not the, highest number of CT scanners per capita in the world. As such, there are exceptionally strong CT users in the cardiac and noncardiac arena, and there are a high number of "stateof-the-art" scanners that exceed the specifications of "64-generation" scanners. Among those "post-64" era technologies [2] featured were 320-detector row CT (Toshiba Aquilion One) and iterative reconstruction to increase spatial resolution and/or lower patient radiation dose (General Electric Discovery 750HD).

Novel uses of 320-detector row CT [3, 4] were described in detail, including applications for perfusion CT, coronary contrast opacification gradients [5], and CT estimations of endothelial shear stress. Dr. DeFrance, in his lecture titled, "CT Stress Myocardial Perfusion-Where are we?" offered his 
perspective on the potential implementation of perfusion CT in the work-up of patients with suspected coronary artery disease, and presented new data acquired at Monash Medical Centre, Victoria, Australia, supporting by way of test characteristics the use of perfusion CT using a comparison with fractional flow reserve obtained at coronary catheterization. This comparison standard for a myocardial perfusion deficit is innovative because it captures highly valuable features of the coronary arteries as well as functional data to determine the hemodynamic significance of coronary lesions. The 320-detector row CT protocol used by the Monash investigators used stress imaging followed by rest images, roughly paralleling the protocol of multiple other studies in cardiac perfusion, including work from Blankstein and colleagues [6]. The lecture and question/answer offered considerable discussion regarding the pros and cons of this approach versus that rest followed by adenosine stress used in the ongoing Core 320 trial. To our knowledge, to date there is little discussion in the literature regard the pros and cons of CT perfusion protocol strategies; submissions of data would be a value to the readership of the International Journal of Cardiovascular Imaging.

Regarding Core320, a multi-center trial with four sites (refer article note) in Japan, several interesting ancillary hypotheses were presented. First, it is essential to study patient radiation doses delivered by perfusion CT to test the hypothesis that the dose delivered by a CT driven algorithm is no greater than that of an algorithm that uses nuclear techniques such as myocardial single photon emission computed tomography (SPECT). While radiation doses for SPECT vary widely and largely depend on the radiotracer administered, to date radiation doses for perfusion CT have been consistently equivalent or low relative to SPECT counterparts, in keeping with the ALARA principle for dose optimization. If any CT perfusion protocol is to become a clinical success, the population radiation dose estimates should be no greater than that those currently delivered, unless any added information is appropriately weighed against the risks of the additional radiation exposure.

An additional hypothesis to be tested is the potential correlation between coronary contrast opacification gradients and lesions considered hemodynamically significant by standard testing (e.g. SPECT, fractional flow reserve, or both). For wide-area detector CT, the gradient is defined as a difference in Hounsfield Units between two points in a coronary artery divided by a normalization factor. If the normalization factor is the distance from the coronary osteum, a so-called distance gradient is obtained. To date, 320-detector row $\mathrm{CT}$ gradients have been shown to be reproducible in normal coronary arteries, likely because of the temporal uniformity inherent in this technology. Preliminary work suggests that vessels with lesions have larger gradients than their normal counterparts; comprehensive evaluation will require data regarding function and clinical outcomes. Coronary wall shear stress [7], also called endothelial shear stress [8,9] can also be estimated using CT. The wall stress is intimately related to the local development of at risk lesions, with low and very low endothelial shear stress states being most concerning. The advantages of newer CT technology include faster scanning, leading to fewer artifacts in these mathematical estimates. Work to date has been promising but challenges remain, including the computation burden, challenges in estimating blood flow (e.g. from bolus tracking data), and the spatial resolution of the technology.

In summary, the array of presentations at the 4th annual meeting of the Japan Regional Committee of the Society of Cardiovascular Computed Tomography was a compelling a comprehensive study of modern cardiac CT that included interesting new applications, novel educational opportunities, and research topics in a format amenable for rapid learning and thoughtprovoking conversation. Much of the complexity from the new CT systems was explained and analyzed at the highest level, and presentations featured new, important applications enabled by CT imaging. The International Journal of Cardiovascular Imaging supports and welcomes submissions from this highly talented group of investigators.

Acknowledgments The authors gratefully acknowledge the assistance from Drs. Shigeyoshi Soga and Noriko Oyama.

\section{References}

1. Rybicki F (2009) Lower radiation dose coronary CT angiography with new imaging technologies. Int $\mathrm{J}$ Cardiovasc Imaging 25:149-151

2. Otero HJ, Steigner ML, Rybicki FJ (2009) The "post-64" era of coronary CT angiography: understanding new technology from physical principles. Radiol Clin North Am 47:79-90 
3. Rybicki FJ, Otero HJ, Steigner ML et al (2008) Initial evaluation of coronary images from 320-detector row computed tomography. Int J Cardiovasc Imaging 24:535-546

4. Steigner ML, Otero HJ, Cai T et al (2009) Narrowing the phase window width in prospectively ECG-gated single heart beat 320-detector row coronary CT angiography. Int J Cardiovasc Imaging 25:85-90

5. Steigner ML, Mitsouras D, Whitmore AG et al (2010) Iodinated contrast opacification gradients in normal coronary arteries imaged with prospectively ECG-gated single heart beat 320-detector row computed tomography. Circ Cardiovasc Imaging 3:179-186

6. Blankstein R, Okada D, Rocha-Filho J et al (2009) Cardiac myocardial perfusion imaging using dual source computed tomography. Int J Cardiovasc Imaging 25:209-216
7. Van der Giessen A, Schaap M, Gijsen F et al. (2010) 3D fusion of intravascular ultrasound and coronary computed tomography for in vivo wall shear stress analysis: a feasibility study. Int J Cardiovasc Imaging 26:781-796

8. Rybicki F, Melchionna S, Mitsouras D et al (2009) Prediction of coronary artery plaque progression and potential rupture from 320-detector row prospectively ECG-gated single heart beat CT angiography: Lattice Boltzmann evaluation of endothelial shear stress. Int J Cardiovasc Imaging 25:289-299

9. Ramkumar PG, Mitsouras D, Feldman CL et al (2009) New advances in cardiac computed tomography. Curr Opin Cardiol 24:596-603 